\title{
An Infection Model of Apple White Rot Based on Conidial Germination and Appressorium Formation of Botryosphaeria dothidea
}

\author{
Ki Woo Kim¹, Kyu Rang Kim² and Eun Woo Park* \\ ${ }^{1}$ National Instrumentation Center for Environmental Management, Seoul National University, Seoul 151-921, Korea \\ ${ }^{2}$ Department of Agricultural Biotechnology, Seoul National University, Seoul 151-921, Korea \\ (Received on October 14, 2005; Accepted on November 18, 2005)
}

Regression models for determining infection periods of apple white rot were developed based on conidial germination and appressorium formation of Botryosphaeria dothidea. A total of 120 apple fruits were inoculated with the fungal conidial suspension and subjected to 6 temperatures and 10 wetness periods. Conidia germinated and produced appressoria, exhibiting swollen tips of germ tubes on the fruit surface. Conidial germination (G) increased with temperature $(T)$ and wetness period (W), and was described as $G=-89.273+7.649 \mathrm{~T}+$ 7.056W $-0.109 T^{2}-0.085 W^{2}-0.066 T W\left(R^{2}=0.75\right)$. Less than 2 hr of wetness period were enough for conidia to germinate at 25 to $30^{\circ} \mathrm{C}$. Effects of temperature and wetness period on appressorium formation (A) could be explained as $A=-1.540-2.375 W+0.045 W^{2}+0.213 T W$ $\left(R^{2}=0.77\right)$. The relationship between conidial germination and appressorium formation $\left(A_{g}\right)$ was described as $A_{\mathrm{g}}=0.381-0.227 \mathrm{G}+0.005 \mathrm{G}^{2}\left(R^{2}=0.67\right)$, suggesting that conidial germination may have to reach approximately $43.7 \%$ to initiate appressorium formation. Using the regression equation for conidial germination and the criterion of $\mathbf{4 3 . 7} \%$ conidial germination, an infection model was developed to determine infection periods based on temperature and wetness period. The infection model with the criterion of $43.7 \%$ conidial germination was apparently more conservative than the appressorium formation model in determining possibility of apple infection. The infection model seemed sensitive to variable weather conditions, suggesting possible use of the model for timing fungicide sprays to control white rot of apples in practice.

Keywords : apple, appressorium, Botryosphaeria dothidea, germination, white rot

Botryosphaeria dothidea Ces. \& De Not. (synonymous with $B$. ribis Grossenb. \& Dug.) is found in most tropical and warm temperate regions as a pathogen of many plants (McGlohon, 1982; Smith, 1934). The fungus belongs to

\footnotetext{
*Corresponding author.

Phone) +82-2-880-4672, FAX) +82-2-873-2317

E-mail) ewpark@snu.ac.kr
}

Ascomycota, and its anamorph is Fusicoccum aesculi Corda (Punithalingam and Holliday, 1973). It causes white rot, one of the most destructive apple (Malus domestica Borkh.) diseases in the world (Sutton, 1990). In Korea, the disease has been a serious problem since the introduction of Fuji, a highly susceptible cultivar to the disease, from Japan in early 1970's (Kim and Kim, 1989; Lee et al., 1993). The disease results in soft and light-colored rot on fruit, and extensive cankers on limbs, eventually resulting in tree death. The main inoculum of $B$. dothidea is conidia, which are present in orchards throughout the growing season of apple fruit (Kim et al., 1995; Sutton and Arauz, 1981). The fungus forms appressoria, that are directly involved in host penetration and adherence to host surfaces, on the fruit surface or enters fruit through natural openings such as lenticels and surface cracks (Kim et al., 1999). Apple fruit can be infected by conidia within 7 weeks after petal fall, and symptoms usually become noticeable 6 to 8 weeks before harvest (Parker and Sutton, 1993b). Ultrastructural aspects of the latent infections and fungal survival were reported (Kim et al., 2001, 2004).

The control of apple white rot relies mainly on chemical practices by periodic fungicide sprays (Sutton, 1990). Season-long benomyl spray at 2-week-intervals was known to be an effective measure for reducing the disease development (Brown and Britton, 1986; Sutton, 1990). Kohn and Hendrix (1983) suggested initiating preventive fungicide sprays when sugar content level reached $10.5 \%$ of apple fruits. However, improper timing of fungicide sprays can result in a considerable yield loss in spite of a full-season protection program. Timing of fungicide sprays can be improved through a better understanding of factors that influence infection processes of the pathogen. Both temperature and wetness period apparently play critical roles in the growth of fungal pathogens and development of various diseases (Arauz and Sutton, 1989; Broome et al., 1995; Jones, 1986; Michailides and Morgan, 1992; Yun and Park, 1990). In order to effectively manage the disease by proper timing of fungicide applications, it is crucial that the effects of temperature and wetness period on the fungal growth are quantitatively described. Then, such a predictive model can 
be used as part of a disease forecasting model (Arauz et al., 1990).

Several infection models have been developed to predict the development of apple white rot. A model made by Kohn and Hendrix (1983) was to predict fruit rot incidence using sugar content of apple fruits throughout the growing season. However, sugar content level fluctuates throughout the growing season, so that it was not assumed to be a reliable indicator of fruit susceptibility (Parker and Sutton, 1993b). Two disease models developed by Parker and Sutton (1993a) were derived from the effect of temperature and wetness period on symptom development on apple fruit pieces. In their study, one model was modified from the other by eliminating additional lesion quantity due to latent infections that occurred prior to fruit sampling in an orchard, which often cause overestimation of infections in artificial inoculation experiments. In order to make a model unaffected by latent infections, parameters involved in the fungal pre-penetration behavior can be employed for model development. Either conidial germination or appressorium formation might be suitable for this purpose, as they are crucial steps on host surface for disease development. Little information is available on the interaction of temperature and wetness period with regard to conidial germination and appressorium formation of $B$. dothidea. The objectives of this study were i) to determine effects of temperature and wetness period on conidial germination and appressorium formation of $B$. dothidea and ii) to develop an infection model for determining infection periods of apple white rot based on hourly temperature and wetness data. Sensitivity of the infection model to weather conditions was compared with a disease model previously developed by Parker and Sutton (1993a) using four sets of weather data obtained from different orchards.

\section{Materials and Methods}

Inoculum preparation. An isolate (BD-3) of $B$. dothidea was obtained from a naturally infected apple fruit exhibiting typical white rot symptoms. The isolate was grown on potato sucrose agar (PSA) slants at $25^{\circ} \mathrm{C}$ for 2 weeks. The culture surface on the PSA slants was flooded with $10 \mathrm{ml}$ of sterilized distilled water and scraped to make a mycelial suspension. The suspension was spread evenly with a sterile bent-glass rod over the surface of freshly prepared acidified $(0.2 \%$ lactic acid) PSA plates amended with streptomycin sulfate at $100 \mu \mathrm{g} / \mathrm{ml}$ to inhibit bacterial contamination. The fungus was then incubated at $27^{\circ} \mathrm{C}$ under continuous fluorescent light with a 12-hr photoperiod for 3 weeks. Pycnidia of the fungus were collected by flooding the plates with sterilized distilled water and scraping the surface of the culture plates. Conidia were dislodged by disrupting pycnidia in sterilized distilled water with a mortar and pestle. The suspension was filtered through 2 layers of cheesecloth to remove mycelial fragments. Inoculum concentration of the conidial suspension was adjusted to $1.0 \times 10^{5}$ conidia/ml using a hemacytometer.

Inoculation of apple fruit. A total of 120 asymptomatic apple (cultivar Fuji) fruits at full maturity were carefully harvested to avoid bruising in an apple orchard at Anseong, Korea. The fruits were washed with mild detergent to remove dirt, insect honeydew, and pesticide residues. They were then surface-sterilized with $0.1 \%$ sodium hypochlorite solution for $2 \mathrm{~min}$, rinsed with tap water, and allowed to air dry. The fruits were inoculated to runoff with the conidial suspension using a hand-held sprayer. Immediately after inoculation, the fruits were placed in a plastic tray $(32 \times 43$ $\times 8 \mathrm{~cm}$ ) lined with moist paper towels, and put into a polyethylene bag for maintaining wetness throughout treatment periods. They were placed in each of 6 incubators at $10,15,20,25,30$, and $35^{\circ} \mathrm{C}$. After the assigned 10 wetness periods of $2,4,8,12,16,20,24,32,40$, and $48 \mathrm{hr}$ at each temperature, 2 fruits were removed for each combination of temperature and wetness period treatments to examine conidial germination and appressorium formation on the fruit surface. The wetness period in this study was defined by the length of time that the inoculum droplet remained as free water on the fruit surface. The wetness treatments were conducted under continuous complete darkness at each temperature.

Specimen preparation for light microscopy. Ten squares of epidermal peels (each $1 \times 1 \mathrm{~cm}$ with approximately 1 $\mathrm{mm}$ thickness of underlying tissue) were excised from the inoculated fruits using a sterile razor blade. The epidermal peels were dipped in EtOH-chloroform $(75: 25, \mathrm{v} / \mathrm{v}) \mathrm{mix}-$ ture amended with trichloroacetic acid at $0.15 \%(\mathrm{w} / \mathrm{v})$ to prevent further conidial germination and decolorize specimens (Wolf and Fric, 1981). The peels were then immersed in $10 \mathrm{ml}$ of distilled water amended with 2 drops (approximately $50 \mu \mathrm{l}$ ) of $0.1 \%$ lactophenol-cotton blue, stained for $10 \mathrm{~min}$, and examined using a light microscope $(\mathrm{BH}$; Olympus Optical, Tokyo, Japan). A conidium was considered germinated if the germ tube was at least one-half the length of the conidium. Percent conidial germination was determined by examining 10 conidia selected randomly on each epidermal peel, a total of 200 conidia from 20 epidermal peels of 2 fruits for a combination of temperature and wetness period treatment. Percent appressorium formation was the number of appressorium-formed conidia out of the germinated conidia.

Model development and sensitivity test. Two multiple 
regression models describing effects of temperature and wetness period on conidial germination and appressorium formation of $B$. dothidea on apple fruits were developed. The quantitative relationship between conidial germination and appressorium formation was also determined. The stepwise procedure was used to select the best set of independent variables using SAS PROC GLM (SAS Institute, Cary, NC). Criteria for selecting the best statistical model included the significance of the $F$ value and coefficient of determination $\left(R^{2}\right)$.

Regression models were tested for their sensitivity in response to four sets of weather data collected from apple orchards in 1993, 1994, 1996, and 2003. For comparison, a disease model which was previously developed by Parker and Sutton (1993a) based on the results of laboratorial experiments determining effects of temperature and wetness period on symptom appearance on apple fruit pieces was also evaluated for its sensitivity to the same weather data sets. The Parker and Sutton's disease model is expressed as follows:

$$
\begin{aligned}
\mathrm{D}= & -0.1546+0.0123 \mathrm{~T}+0.0329 \mathrm{~W}-0.00169 \mathrm{~W}^{2} \\
& +0.0000225 \mathrm{~W}^{3}-0.00153 \mathrm{TW}+0.000111 \mathrm{TW}^{2} \\
& -0.00000151 \mathrm{TW}^{3}
\end{aligned}
$$

where $\mathrm{D}$ is the percentages of diseased fruit pieces. $\mathrm{T}$ and $\mathrm{W}$ are hourly temperature $\left({ }^{\circ} \mathrm{C}\right)$ and wetness period $(\mathrm{hr})$, respectively.

Each weather data set for the model sensitivity tests consisted of hourly temperature and wetness period from June 1 to September 30. The 4-year microclimatic data were collected using automated weather stations installed in apple orchards at 4 different locations in Korea; i) Chonan in 1993, ii) Suwon in 1994, iii) Pyongtaek in 1996, and iv) Naju in 2003. The automated weather stations consisted of a datalogger (CR10; Campbell Scientific, USA), temperature (Model 107; Campbell Scientific, USA) and leaf wetness sensors (Model 237; Campbell Scientific, USA). In order to avoid overestimation of the infection risk, the simulation was performed to end infection periods by interrupted wetness period based on the results of $B$. obtusa, a closely related species of $B$. dothidea, causing apple black rot (Arauz and Sutton, 1990). The model sensitivity was evaluated in terms of the amount of variations in duration of daily infection periods (hr) over the test periods. As for the Parker and Sutton's model, the amount of variations in daily percentages of diseased pieces over the test periods was used as the indicator of model sensitivity.

\section{Results}

Conidial germination. Conidia of $B$. dothidea were fusoid and straight in shape. Conidia germinated and produced

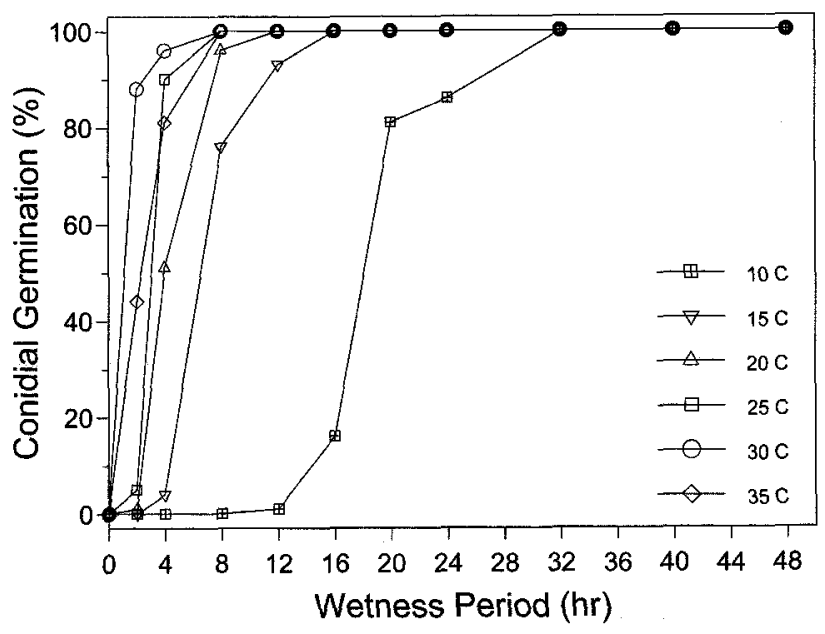

Fig. 1. Effects of temperature and wetness period on conidial germination of $B$. dothidea on apple fruits.

germ tubes from either one or both ends of conidia. There was active conidial germination even in short wetness periods on the fruit surface (Fig. 1). Approximately 31, 88, and $44 \%$ of conidia germinated after $2 \mathrm{hr}$ at 25,30 , and $35^{\circ} \mathrm{C}$, respectively. After $8 \mathrm{hr}$, all the inoculated conidia germinated at 25,30 , and $35^{\circ} \mathrm{C}$. The optimum temperature for conidial germination of $B$. dothidea ranged from 25 to $30^{\circ} \mathrm{C}$. Lower temperatures required longer wetness periods for conidial germination. Only $17 \%$ of conidia germinated at $10^{\circ} \mathrm{C}$ even after $16 \mathrm{hr}$ of wetness period.

Appressorium formation. Conidial germ tubes grew on the fruit surface and formed appressoria, exhibiting swollen tips of germ tubes. Percent appressorium formation increased with temperature up to $30^{\circ} \mathrm{C}$ as in conidial germination (Fig. 2). Appressorium formation required longer than $2 \mathrm{hr}$

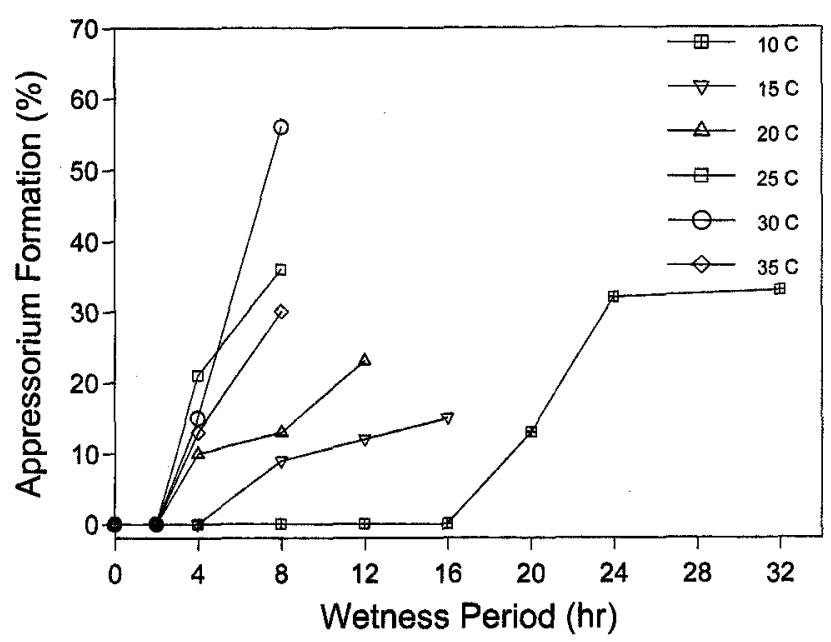

Fig. 2. Effects of temperature and wetness period on appressorium formation of $B$. dothidea on apple fruits. 


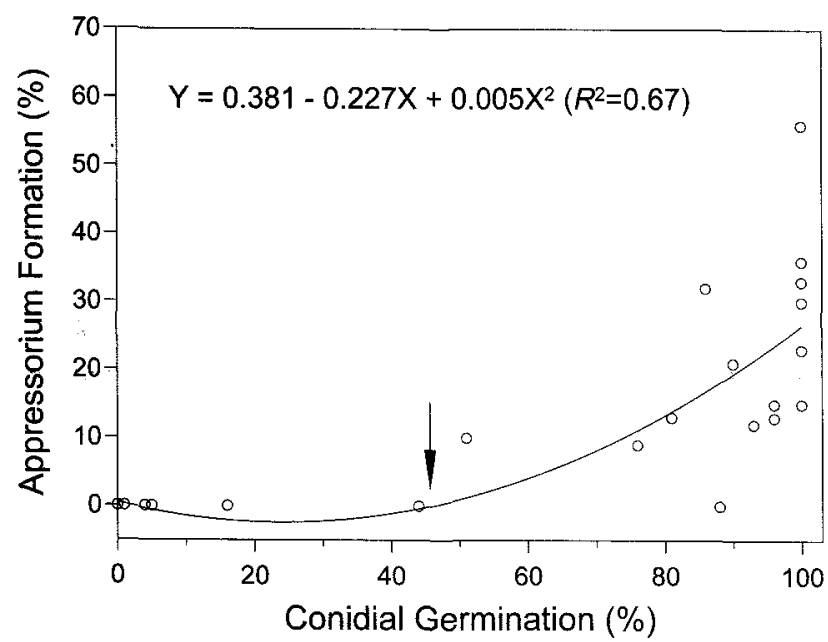

Fig. 3. Relationship between conidial germination and appressorium formation of $B$. dothidea. Appressorium formation was initiated when conidial germination reached approximately $43.7 \%$ (an arrow) at a given temperature and wetness period.

of wetness period even at $25^{\circ} \mathrm{C}$. After $8 \mathrm{hr}, 36,56$, and $30 \%$ of germinated conidia produced appressoria at 25,30, and $35^{\circ} \mathrm{C}$, respectively. Longer than $16 \mathrm{hr}$ of continuous wetness were required to produce appressoria at $10^{\circ} \mathrm{C}$. The optimum temperature for the fungal appressorium formation ranged from 25 to $30^{\circ} \mathrm{C}$. As appressoria usually formed at the tips of germ tubes grown long from a conidium, some appressoria could not be determined from which conidia they had originated. Consequently, percent appressorium formation could not be determined after $8 \mathrm{hr}$ of wetness at 25,30 , and $35^{\circ} \mathrm{C}, 12 \mathrm{hr}$ at $20^{\circ} \mathrm{C}$, and $16 \mathrm{hr}$ at $15^{\circ} \mathrm{C}$.

Relationship between conidial germination and appressorium formation. Appressorium formation increased as conidial germination increased under continuous wetness periods (Fig. 3). The relationship between conidial germination $(G)$ and appressorium formation $\left(A_{g}\right)$ could be described by the following regression model:

$$
A_{g}=0.381-0.227 \mathrm{G}+0.005 \mathrm{G}^{2}
$$

The coefficient of determination of Eq. (1) was 0.67 , indicating that $67 \%$ of variations in percentage of appressorium formation could be accounted for by conidial germinations. All estimates of parameters were statistically significant $(P=0.01)$. Until conidial germination reached approximately $43.7 \%$ at a given temperature and wetness period, appressorium formation was hardly observed. However thereafter, gradual increase of appressorium formation was evident on germinated conidia.

Model development and sensitivity test. The combined

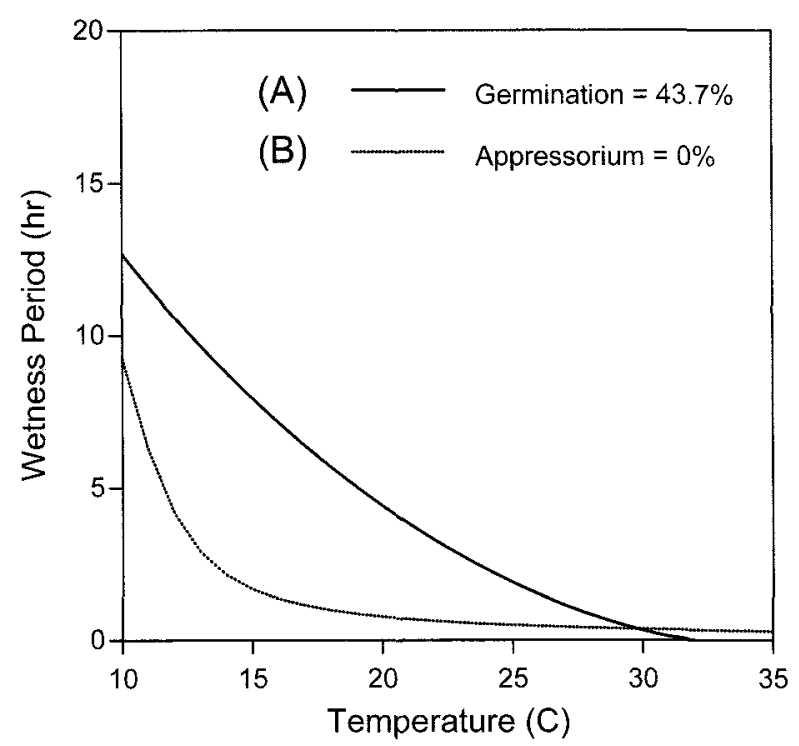

Fig. 4. Critical temperature and wetness period conditions required for (A) $43.7 \%$ conidial germination and (B) appressorium formation of $B$. dothidea.

effects of temperature $(\mathrm{T})$ and wetness period $(\mathrm{W})$ on percent conidial germination $(\mathrm{G})$ and appressorium formation (A) could be described by the following regression models:

$$
\begin{aligned}
\mathrm{G}= & -89.273+7.649 \mathrm{~T}+7.056 \mathrm{~W}-0.109 \mathrm{~T}^{2}-0.085 \mathrm{~W}^{2} \\
& -0.066 \mathrm{TW} \\
\mathrm{A}= & -1.540-2.375 \mathrm{~W}+0.045 \mathrm{~W}^{2}+0.213 \mathrm{TW}
\end{aligned}
$$

The coefficients of determination of Eqs. (2) and (3) were 0.75 and 0.77 , respectively, indicating that $75 \%$ and $77 \%$ of variations in percentages of conidial germination and appressorium formation, respectively, could be accounted for by temperature and wetness period. All estimates of parameters were statistically significant $(P=0.01)$.

From Eqs. (2) and (3), wetness periods required for $43.7 \%$ conidia to germinate and appressoria to form at certain temperatures were calculated by substituting $\mathrm{G}$ in Eq. (2) and A in Eq. (3) with 43.7 and 0, respectively (Fig. 4). According to the relationship between conidial germination and appressorium formation in Fig. 3, appressorium formation was hardly initiated on the surface of apple fruits until conidial germination reached approximately $43.7 \%$ at a given temperature. Therefore, both graphs in Fig. 4 determine the temperature and wetness period conditions under which $B$. dothidea could form appressoria to infect apple fruits. Eq. (2) with the criterion of $43.7 \%$ conidial germination was apparently more conservative than Eq. (3) in determining possibility of apple infection. In this study, we decided to use Eq. (2) with the criterion of $43.7 \%$ conidial germination as the model to determine infection 
Table 1. Comparison of sensitivity of two infection models of apple white rot using four sets of weather data obtained from different orchards

\begin{tabular}{cccc}
\hline \multirow{2}{*}{ Location } & Year & \multicolumn{2}{c}{ Variance of daily model output $^{\mathrm{a}}$} \\
\cline { 3 - 4 } & & Infection model $^{\mathrm{b}}$ & $\begin{array}{c}\text { Parker and Sutton's } \\
\text { disease model }^{\mathrm{c}}\end{array}$ \\
\hline Chonan & 1993 & 86.83 & 49.94 \\
Suwon & 1994 & 38.44 & 34.74 \\
Pyongtaek & 1996 & 90.69 & 68.71 \\
Naju & 2003 & 119.46 & 80.98 \\
\hline
\end{tabular}

${ }^{a}$ Variances of daily output values generated by the models using daily temperature and wetness period data from June 1 to September 30 .

${ }^{b}$ The infection model developed in this study is based on the effects of temperature and wetness period on conidial germination with $43.7 \%$ conidial germination as the appressorium formation. Data are variances of daily infection periods (hr) determined by the model.

${ }^{\mathrm{c}}$ A disease model previously developed by Parker and Sutton (1993a). Data are variances of daily percent disease fruit pieces determined by the model.

periods of the fungus. For clarity in the text, this model is called 'the infection model' hereafter.

Using four weather data sets collected from apple orchards, the daily infection periods in hours and the percentage of daily diseased fruit pieces were calculated from the infection model and the Parker and Sutton's disease model, respectively. Since two models generate outputs in different units, their sensitivity in response to variable weather conditions was compared in terms of variances of daily outputs from the models (Table 1). The variance of daily infection periods determined by the infection model developed in this study was always greater than that of daily diseased fruit pieces determined by the disease model developed by Parker and Sutton (1993a), indicating that the infection model responded more sensitively to weather conditions than the Parker and Sutton's disease model.

\section{Discussion}

This study demonstrated that temperature and wetness period directly affected conidial germination and appressorium formation of $B$. dothidea. Both conidial germination and appressorium formation increased at high temperatures ( 25 to $30^{\circ} \mathrm{C}$ ) within less than two hours of wetting on the surface of apple fruits. Furthermore, $B$. dothidea produces conidia over the 6 to $30^{\circ} \mathrm{C}$ range with a maximum sporulation at 18 to $24^{\circ} \mathrm{C}$ (Copes and Hendrix, 2004) and the conidia are primarily discharged by rainwater in summer months (Kim et al., 1995; Sutton and Arauz, 1981). These previous observations and the results from this study suggest that either fungal penetration into host tissues or entrance through natural openings on apple fruits can occur easily during the apple growing seasons in Korea. The responses of conidial germination to temperature in this study are consistent with those obtained by Sutton and Arauz (1991) who found the optimum temperature for conidial germination ranged from 26.7 to $29.5^{\circ} \mathrm{C}$.

Regression models for conidial germination and appressorium formation responding to temperature and wetness period were developed to provide information on possible infection by $B$. dothidea for scheduling fungicide sprays. The infection model developed in this study was derived from the effects of temperature and wetness period on conidial germination on apple fruits, and the quantitative relationship between conidial germination and appressorium formation. Consequently the daily infection period determined by the infection model was the time period in hours being continuously wet after $43.7 \%$ of conidia on the surface of apple fruit germinated in a day.

In comparison with the Parker and Sutton's disease model, the infection model in this study appeared to be relatively sensitive to weather conditions in apple orchards. Although the infection model has not yet been tested for its validity in predicting fungal infections in apple orchards, the relatively high sensitivity of the model in response to variable weather conditions would probably suggest its effectiveness or better performance than the Parker and Sutton's disease model in identifying favorable weather conditions for fungal infection. The small variance of daily infection periods at Suwon in 1994 was attributed to the relatively unfavorable weather conditions due to less frequent rainfall events over the period as compared with other years (data not shown).

The infection model in this study was developed based on the experimental results obtained under continuous wetting conditions. However, interruptions of wetness periods often occurs in cases of nocturnal wetting and diurnal drying cycles, and intermittent rain showers. Therefore, fungal spores and germ tubes should be exposed to alternating wet and dry periods before host infection. It remains to be revealed whether interrupted wetness periods are detrimental to the growth of $B$. dothidea or not. In order to be widely practiced in apple disease management, the infection model should be evaluated for its effectiveness in scheduling fungicide sprays in apple orchards. Comparison of season-long disease progress under predicted and conventional fungicide spray programs would provide information on the improvement of current management strategy of the disease.

\section{Acknowledgments}

This study was supported by the Korea Science and Engineering Foundation (Project Number 91-07-00-06). 


\section{References}

Arauz, L. F. and Sutton, T. B. 1989. Temperature and wetness duration requirements for apple infection by Botryosphaeria obtusa. Phytopathology 79:440-444.

Arauz, L. F. and Sutton, T. B. 1990. Effect of interrupted wetness periods on spore germination and apple infection by Botryosphaeria obtusa. Phytopathology 80:1218-1220.

Arauz, L. F., Sutton, T. B. and Pope, L. R. 1990. Simultaneous use of infection criteria for three apple diseases for timing of fungicide sprays. Phytopathology 80:1212-1218.

Broome, J. C., English, J. T., Marois, J. J., Latorre, B. A. and Aviles, J. C. 1995. Development of an infection model for Botrytis bunch rot of grape based on wetness duration and temperature. Phytopathology 85:97-102.

Brown, E. A., II and Britton, K. O. 1986. Botryosphaeria diseases of apple and peach in the southeastern United States. Plant Dis. 70:480-484.

Copes, W. E. and Hendrix, F. F. Jr. 2004. Effect of temperature on sporulation of Botryosphaeria dothidea, B. obtusa, and $B$. rhodina. Plant Dis. 88:292-296.

Jones, A. L. 1986. Role of wet period in predicting foliar diseases. In: Plant Disease Epidemiology, Population Dynamics and Management, vol. 1, ed. by K. J. Leonard and W. E. Fry, pp. 87-100. Macmillan Publishing, New York, NY, USA.

Kim, S. B. and Kim, C. S. 1989. Pathogenicity and ecology of apple rot caused by Botryosphaeria dothidea. I. Pathogen identification and effects of different media and culture conditions on mycelial growth. J. Kor. Soc. Hort. Sci. 30:26-37. (in Korean)

Kim, K. W., Park, E. W. and Ahn, K.-K. 1999. Pre-penetration behavior of Botryosphaeria dothidea on apple fruits. Plant Pathol. J. 15:223-227.

Kim, K. W., Park, E. W. and Kim, K. S. 2004. Glyoxysomal nature of microbodies complexed with lipid globules in Botryosphaeria dothidea. Phytopathology 94:970-977.

Kim, K. W., Park, E. W., Kim, Y. H., Ahn, K.-K., Kim, P. G. and Kim, K. S. 2001. Latency- and defense-related ultrastructural characteristics of apple fruit tissues infected with Botryosphaeria dothidea. Phytopathology 91:165-172.

Kim, K. W., Park, E. W., Kim, S. B. and Yun, J. I. 1995. Temporal dynamics of Botryosphaeria dothidea spore dispersal in apple orchards and related climatological factors. Korean J. Plant Pathol. 11:230-237 (in Korean).

Kohn, F. C., Jr. and Hendrix, F. F. 1983. Influence of sugar content and $\mathrm{pH}$ on development of white rot on apples. Plant Dis. $67: 410-412$

Lee, Y. H., Cho, W. D., Kim, W. K., Lee, E. J., Han, S. J. and Chung, H. S. 1993. Detailed survey of apple and pear diseases in major fruit producing areas in Korea ('88-'92). Korean J. Plant Pathol. 9:47-51.

McGlohon, N. E. 1982. Botryosphaeria dothidea-Where will it stop? Plant Dis. 66:1202-1203.

Michailides, T. J. and Morgan, D. P. 1992. Effects of temperature and wetness duration on infection of pistachio by Botryosphaeria dothidea and management of disease by reducing duration of irrigation. Phytopathology 82:1399-1406.

Parker, K. C. and Sutton, T. B. 1993a. Effect of temperature and wetness duration on apple fruit infection and eradicant activity of fungicides against Botryosphaeria dothidea. Plant Dis. 77:181-185.

Parker, K. C. and Sutton, T. B. 1993b. Susceptibility of apple fruit to Botryosphaeria dothidea and isolate variation. Plant Dis. 77:385-389.

Punithalingam, E. and Holliday, P. 1973. Botryosphaeria ribis. Descriptions of Pathogenic Fungi and Bacteria, No. 395. Commonwealth Mycological Institute, Kew, Surrey, England.

Smith, C. O. 1934. Inoculations showing the wide host range of Botryosphaeria ribis. J. Agric. Res. 49:467-476.

Sutton, T. B. 1990. White rot. In : Compendium of Apple and Pear Diseases, ed. by A. L. Jones and H. S. Aldwinckle, pp. 16-18. APS Press, St. Paul, MN, USA.

Sutton, T. B. and Arauz, L. F. 1981. Production and dispersal of conidia by Physalospora obtusa and Botryosphaeria dothidea in apple orchards. Phytopathology 71:584-589.

Sutton, T. B. and Arauz, L. F. 1991. Influence of temperature and moisture on germination of ascospores and conidia of Botryosphaeria dothidea. Plant Dis. 75:1146-1149.

Wolf, G. and Fric, F. 1981. A rapid method for staining Erysiphe graminis f. $\mathrm{sp}$. hordei in and on whole barley leaves with a protein-specific dye. Phytopathology 71:596-598.

Yun, S. C. and Park, E. W. 1990. Effects of temperature and wetness period on infection of grape by Colletotrichum gloeosporioides. Korean J. Plant Pathol. 6:219-228. (in Korean) 